\title{
Features Construction Geospatial Web Applications and Services for the Environmental Monitoring Systems
}

\author{
Alexey A. Kadochnikov* \\ Institute of Computational Modeling SB RAS \\ 50/44 Akademgorodok, Krasnoyarsk, 660036, Russia
}

Received 02.03.2015, received in revised form 13.09.2015, accepted 17.10.2015

The paper discusses the features encountered in the development of information-analytical system for environmental monitoring of the natural environment and resources, built on the basis of GIS technologies, Internet, remote sensing data processing and data from monitoring stations. Describes the functionality created software interfaces and web services for data collection, data processing and visualization of observation.

Keywords: spatial data, the natural environment, monitoring, GIS Internet server, web-cartography, GIS.

DOI: $10.17516 / 1999-494 X-2015-8-7-908-916$.

\section{Особенности построения геопространственных веб-приложений и сервисов \\ для систем мониторинга состояния окружающей природной среды}

А.А. Кадочников

Институт вычислительного моделирования СО РАН Россия, 660036, Красноярск, Академгородок, 50/44

Рассматриваются особенности, возникающие при разработке информационноаналитических систем для экологического мониторинга состояния природной среды и ресурсов, построенных на основе технологий ГИС, Интернет, обработки данных дистанционного зондирования и данных со станций наблюдения. Описаны функциональные возможности созданных программных интерфейсов и веб-сервисов для сбора, обработки и визуализации данных наблюдения.

Ключевые слова: пространственные данные, окружающая среда, система мониторинга, геоинформационный интернет-сервер, веб-картография, ГИС.

(C) Siberian Federal University. All rights reserved

* Corresponding author E-mail address: scorant@icm.krasn.ru 


\section{Введение}

С одной стороны, мониторинг состояния окружающей природной среды в зоне действия различных промышленных предприятий позволяет сократить расходы на ликвидацию последствий техногенных аварий, что, в свою очередь, снижает вероятность загрязнения почвы, поверхностных вод, гибели растительности и представителей животного мира. С другой стороны, протяжённая территория Красноярского края, климатическое, ландшафтное природноресурсное разнообразие диктуют необходимость применения пространственных методов и средств анализа в задачах мониторинга качества природной среды и использования ее ресурсов. Уровень развития современных геоинформационных технологий дает возможность использовать их и как место для хранения, обработки и презентации тематических данных мониторинга, и как инструмент анализа качества природной среды.

Рассматривается задача создания информационно-аналитических систем для экологического мониторинга состояния природной среды и ресурсов, построенных на основе технологий ГИС, Интернет, обработки данных дистанционного зондирования и данных со станций наблюдения. Значительное внимание уделяется веб-сервисам и программным интерфейсам. В работе важную роль играет использование современных средств визуализации данных с помощью ГИС-технологий [1]. В таких задачах использование интернет-технологий имеет ряд преимуществ по сравнению с настольными ГИС - доступность предлагаемых решений большому числу пользователей, упрощение процесса установки и распространения программного обеспечения, снижение его стоимости, возможность интеграции со сторонними приложениями и проч. [2].

\section{Распределенное хранилище пространственных данных и метаданных}

В качестве основы для сбора, обработки и визуализации данных наблюдения за окружающей средой применяются разработки коллектива Института вычислительного моделирования СО РАН (ИВМ СО РАН) - геопортал института. Геопортал представляет собой распределенное хранилище пространственных данных и метаданных, программные средства для анализа пространственных данных с использованием технологий, предлагаемых международным консорциумом Open Geospatial Consortium (OGC, http://www.opengeospatial.org) и программного обеспечения MapServer (http://mapserver.org/) и GeoWebCache (http://geowebcache.org/). Программные инструменты содержат средства для хранения цифровых картографических материалов, растровых снимков территории, сервисы для навигации по распределенному каталогу пространственных данных, сервисы для пространственного анализа. Основным элементом геопортала является каталог метаданных о пространственных данных.

Каталог метаданных содержит информацию по доступным слоям, картам и другим ресурсам. Основной особенностью каталога пространственных данных является возможность использования различных форматов пространственных данных и организация доступа для пользователя к этим данным с помощью современных стандартов и технологий. Для оформления карт и картографических слоев применяется Styled Layer Descriptor (SLD) - язык описания стилей, используемый для отображения объектов на карте в Web Map Service (WMS) и Web Feature Service (WFS) серверах, а также собственный формат

$$
-909-
$$


описания стилей, разработанный для геопортала ИВМ СО РАН. Описание стиля содержит информацию о визуальном оформлении объектов картографического слоя в зависимости от настроек видимости, включающих ограничение на масштабы видимости, типы объектов и фильтры по атрибутивным данным. Существуют возможности гибкой настройки отображения подписей объектов.

В работе важную роль играет система сбора оперативных данных наблюдения от различных веб-сервисов, станций наблюдения и датчиков. В рамках геопортала ИВМ СО РАН был разработан блок для сбора, обработки и представления данных различных наблюдений [3]. Организация доступа к данным наблюдений осуществляется стандартными средствами геопортала, включающего просмотр табличных данных, экспорт, просмотр данных на картах с возможностью выбора временных интервалов и доступ с помощью общепринятых стандартов. Организован доступ к собранным данным оперативного мониторинга с помощью стандарта Sensor Observation Service (SOS), разрабатываемого консорциумом OGC.

\section{Программно-аппаратная платформа}

Для построения клиентской части веб-приложения, использующего карту региона, были проанализированы два способа представления картографической информации для пользователя. Первый способ - карта отображается с помощью фрагментов. Эту технологию используют такие ресурсы, как Google Maps (http://maps.google.com), Яндекс Карты (http://maps.yandex. ru), Карты Bing (http://www.bing.com/maps/) и др. Основным преимуществом такого способа является скорость получения визуальной информации пользователем и малая нагрузка на сервер при отображении статической информации. Процесс формирования карты на клиентском компьютере состоит из нескольких этапов с применением дополнительных программных потоков, механизма кэширования, очереди загрузки фрагментов и др. При таком способе отображения карты процесс построения композиции карты позволяет пользователю оптимизировать процесс загрузки, снизить нагрузку на веб-браузер и более равномерно ее распределить по времени. Однако при отображении меняющихся тематических данных, необходимых для информационно-аналитических систем, такой способ снижает скорость доступа пользователя к пространственным данным и увеличивает нагрузку на сервер. Для решения этой проблемы обращаются ко второму способу отображения информации - по запросу пользователя генерируется одно растровое изображение либо формируется слой с векторными объектами. В зависимости от типа представляемой информации пользователю в программном интерфейсе системы используется комбинация этих двух способов.

Сегодня существует большое число библиотек с открытым исходным кодом для создания готового пользовательского интерфейса с картографическим интерфейсом, например, OpenLayers (http://www.openlayers.org), LeafLet (http://leafletjs.com/), GeoExt (http://geoext.org), Fusion (http://trac.osgeo.org/fusion/) и др. Однако функционала существующих библиотек недостаточно для решения поставленной задачи и было разработано веб-приложение с использованием библиотеки OpenLayers. OpenLayers - это JavaScript-библиотека с открытым исходным кодом, предназначенная для создания карт на основе программного интерфейса, подобного API Яндекс.Карт (http://api.yandex.ru/maps/) или GoogleMap API (http://code.google.com/intl/ru/ apis/maps/), поддерживает технологию АЈАХ и анимацию.

$$
-910-
$$


При разработке серверной части веб-приложения для работы с пространственными данными используется программное обеспечение MapServer, предназначенное для обеспечения доступа через Интернет к интерактивным картам, различной пространственно увязанной информации. MapServer представляет собой открытую и свободно распространяемую среду разработки интернет-приложений для работы с электронными картами широко распространенных среди множества геоинформационных систем векторных и растровых форматов, обладающую большим числом функциональных возможностей.

Для создания карты из растровых фрагментов обращались к программному обеспечению GeoWebCache. GeoWebCache использует спецификацию WMS Tile Caching (WMS-C) [4], которая явилась результатом конференции FOSS4G в 2006 г. Серверы WMS-C по протоколам совместимы с OGC WMS, поэтому их можно встроить между клиентом и сервером WMS, что позволяет существенно увеличить скорость реакции и разгрузить сервер. Рассмотрены альтернативные решения для создания каталога фрагментов (тайлов), такие как ka-Map Cache (http:// ka-map.ominiverdi.org), TileCache (http://tilecache.org), MapCache (http://mapserver.org) и др. Источником пространственных данных для сервера с программным обеспечением GeoWebCache послужил WMS-сервер с картой Красноярского края на основе программного обеспечения MapServer. Реализована система сервисов, которые поддерживают кэш растровых изображений на сервере с GeoWebCache в актуальном состоянии при обновлении исходных данных на WMS-сервере.

В результате объединения различных технологий представления карты пользователю на стороне клиента реализован вариант, в котором карта состоит из двух слоев: карта-подложка и тематический слой.

Отдельное внимание в работе уделено созданию карты подложки для отображения различных тематических данных на территории Красноярского края, включая данные наблюдения. Большинство доступных карт на основе растровых фрагментов в сети Интернет представлены в цилиндрической прямой равноугольной проекции Меркатора на шаре (код EPSG:3857, или в старом варианте EPSG:900913). Эта проекция используется в таких сервисах, как Google Maps, OpenStreetMap и др. В Яндекс Картах используется цилиндрическая прямая равноугольная проекция Меркатора на эллипсоиде WGS 84 (код EPSG:3395). Также часто применима для отображения геодезическая система координат на эллипсоиде WGS 84 (Широта/Долгота) (код EPSG:4326). Однако эти проекции обладают рядом недостатков при отображении территории Красноярского края. Красноярский край вытянут на карте с юга на север, и при отображении его территории с описанными выше проекциями происходят искажения северных территорий. В свою очередь, основные проекции мелкомасштабных карт России (различные редакции нормальной конической равнопромежуточной, косой перспективно-цилиндрической проекций и проч.) обычно не используются при создании веб-карт - для них не определены коды в системе классификации EPSG, которая является обязательной для работы публичных сервисов на основе стандартов OGC. При просмотре тематических данных поверх карты подложки пользователь не только обращает внимание на стилевое отличие представленных данных, но также соотносит размеры территорий (рис. 1). Для решения этой проблемы были созданы альтернативные карты-подложки с применением проекции, более подходящей для территории Красноярского края. В частности, использовалась система координат 1942 в проекции Гаусса Крю- 

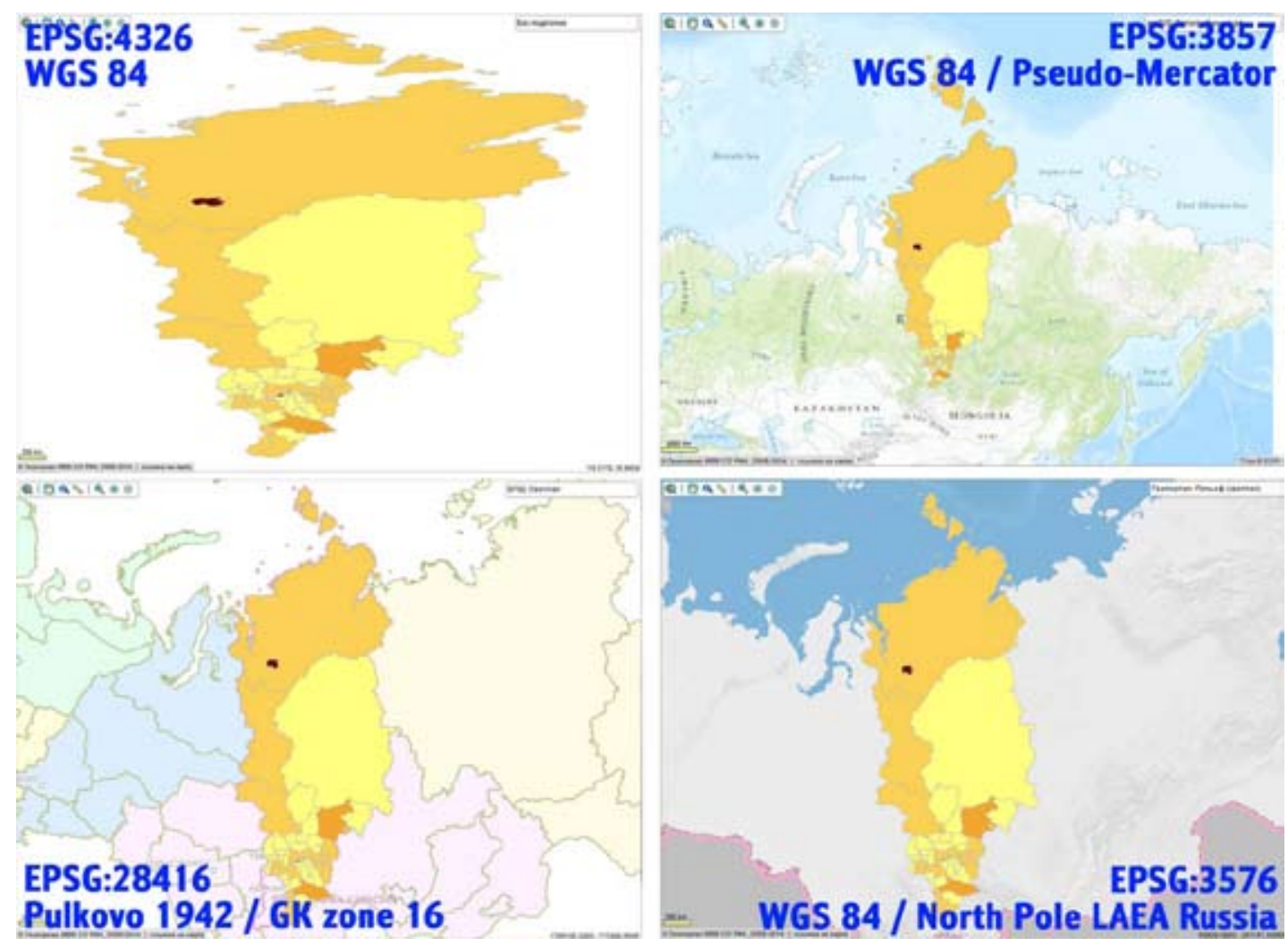

Рис. 1. Выбор проекции карты для визуализации тематических данных

гера (СК 1942) (код EPSG:28416) и азимутальная равновеликая проекция Ламберта (Lambert Azimuthal Equal Area) (код EPSG:3576).

При отображении тематического слоя могут использоваться данные различных наблюдений, полученные от сервиса «Sensor collector API», разработанного сотрудниками ИВМ CO РАН [5]. Для наполнения базы данных наблюдений этого сервиса был подготовлен модуль сбора данных, который включает в себя набор различных «адаптеров» или «драйверов» для различных источников информации (рис. 2). Эти данные периодически загружаются с внешнего источника, который может быть представлен в виде потока информации в форматах txt, csv, json, xml, html и др. Разработанное программное обеспечение извлекает из полученных данных необходимую информацию и загружает с помощью АРI сервиса на сервер. В дальнейшем эти данные могут быть использованы различными способами: доступ к данным может быть организован в виде SOS-сервисов; результаты наблюдений могут быть представлены в виде веб-приложений для дальнейшей визуализации и анализа; данные могут быть переданы для дальнейшей обработки, результат которой, в свою очередь, также может быть представлен пользователю с помощью картографических веб-сервисов. Такие данные могут обрабатывать моментально и на протяжении некоторого продолжительного временного промежутка. В первом случае пользователь видит результат сразу после запроса, во втором - пользователь ожидает результат обработки данных и в дальнейшем может эти данные получить в виде потока 
новых данных. Необходимо отметить, что большие объемы данных при таком подходе могут обрабатываться с помощью вычислительных кластеров.

Источником для модуля сбора данных могут служить: данные других SOS-сервисов; данные различных систем мониторинга; данные станций мониторинга с различными сенсорами; различные архивы наблюдения.

\section{Пример работы системы визуализации данных наблюдения}

В качестве первого примера визуализации данных наблюдения для демонстрации возможности разрабатываемых программных средств взяты данные, полученные от краевой системы наблюдения за состоянием окружающей среды на территории Красноярского края. Поддержкой этой системы наблюдения занимается Краевое государственное бюджетное учреждение «Центр реализации мероприятий по природопользованию и охране окружающей среды Красноярского края». Одним из основных видов деятельности учреждения является обеспечение формирования и согласованного с государственной службой наблюдения функционирования краевой системы наблюдения за состоянием окружающей среды на территории Красноярского края. Наблюдения за качеством атмосферного воздуха проводят на шести автоматизированных постах наблюдений (АПН): в зоне воздействия ОАО «РУСАЛ Ачинск» один АПН - город Ачинск (Юго-Восточный район); в зоне воздействия ОАО «РУСАЛ Красноярск» пять АПН город Красноярск (мкр. Северный, мкр. Солнечный, мкр. Черемушки), пос. Березовка Березовского района, деревня Кубеково Емельяновского района.

АПН представляют собой павильоны, в которых размещено оборудование, обеспечивающее непрерывное автоматическое измерение массовых концентраций в атмосферном воздухе загрязняющих веществ и метеорологических параметров [6]. Для загрузки данных подготовлен программный модуль с соответствующим «драйвером» для обработки и преобразования входных данных, обеспечивающий периодическую загрузку данных наблюдений от этой системы через еe сайт (http://krasecology.ru). На данный момент для просмотра на геопортале доступен следующий набор данных: азота диоксид $\left(м \Gamma / \mathrm{M}^{3}\right)$, азота оксид $\left(\mathrm{M \Gamma} / \mathrm{M}^{3}\right)$, атмосферное давление (мм. рт. ст.), взвешенные вещества (мг/м³), влажность (\%), интенсивность осадков (мм/ч), серы

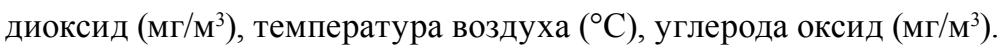

Для публикации данных наблюдения в каталог геопортала был добавлен новый тип ресурса - «данные наблюдения». При публикации такого ресурса пользователю предоставляется ряд настроек, включающий выбор из списка доступных станций наблюдения и сенсоров. Каждая станция наблюдения имеет пространственную привязку и определенный набор сенсоров. При этом разные станции наблюдения, не относящиеся к одной группе, могут иметь несколько общих сенсоров. Для просмотра данных наблюдений были расширены возможности существующего картографического веб-интерфейса для пользователя. Основные элементы нового интерфейса - карта-подложка и данные наблюдения в виде полупрозрачного слоя на выбранный момент времени для выбранного показателя. В левой части веб-приложения пользователю доступны элементы управления, включающие выбор одного из показателей и временного интервала.

На текущем этапе в качестве примера с помощью интерполяции неравномерно распределенных точек в двумерном пространстве автоматически строится поверхность карты с рас- 


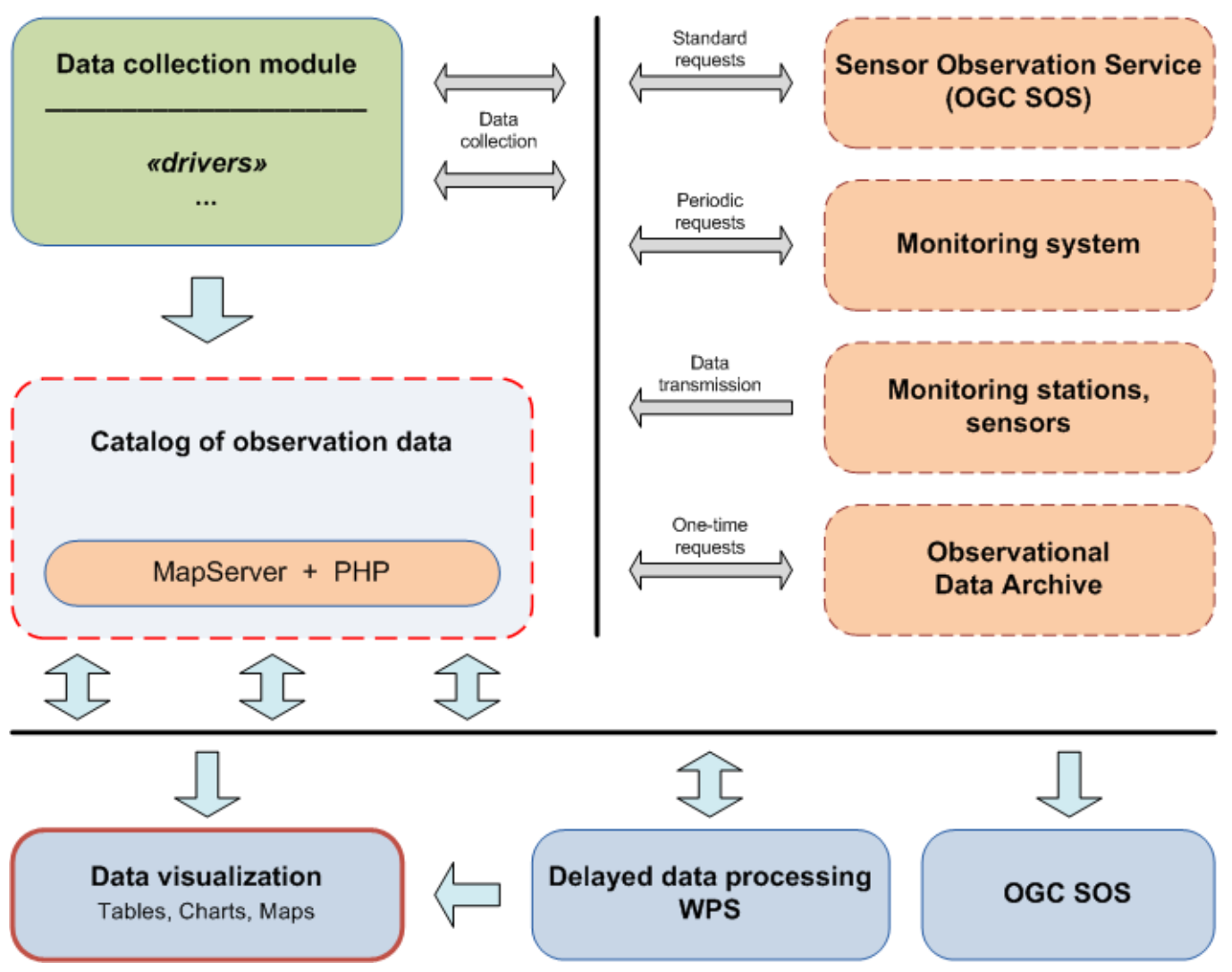

Рис. 2. Обработка и визуализация данных мониторинга

пределением данных наблюдения. Был подготовлен программный модуль для построения сетки распределения данных наблюдения с помощью алгоритма взвешенной усредненной оценки (IDW - Inverse Distance Weighting). При IDW-интерполяции учитываются значения во всех исходных точках, лежащих в пределах заданного радиуса поиска вокруг узла сети (или указанном подмножестве) [7]. Этот алгоритм достаточно быстро работает на небольшой сетке. Тем не менее возникают проблемы при больших объемах данных. Также алгоритм обладает рядом недостатков, что препятствуют использованию алгоритма в большинстве практических задач [8].

Логика разработанного программного решения не ограничивает пользователя в применении реализованного алгоритма. Для построения карты с распределением данных наблюдения могут быть использованы другие алгоритмы. Простые алгоритмы могут быть также реализованы с использованием языка PHP (Hypertext Preprocessor). Более сложные и более требовательные к системе решения могут быть реализованы в виде исполняемых файлов для операционной системы Unix. В дальнейшей работе планируется использовать алгоритмы отложенной обработки для сложных и длительных по времени процессов преобразования данных наблюдений. Для этой цели могут выступать вычислительные кластеры, сервисы, предоставляющие доступ к «большим данным» [9], и различные сервисы WPS, предоставляющие услуги геопроцессинга растровых и векторных данных. 

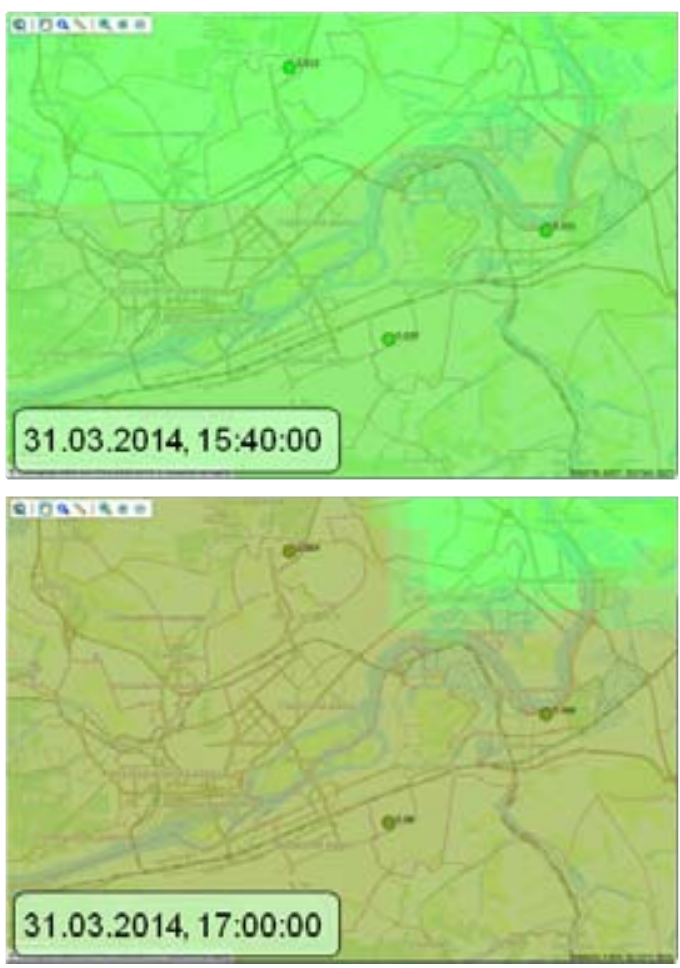
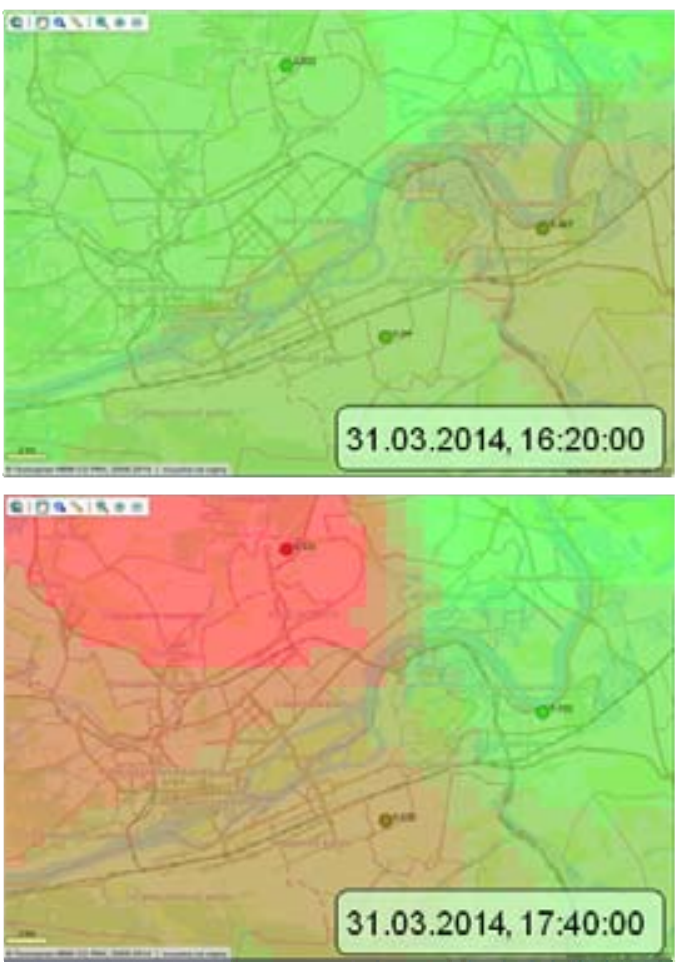

Рис. 3. Пример динамики по времени с данными наблюдения

Необходимо отметить, что при применении небольшой сетки (20 на 20) рационально было использовать вариант отображения данных на стороне клиента в виде векторных объектов. Такой подход позволял просматривать динамику по времени с малой нагрузкой на интернетканал. Однако при увеличении размера сетки возрастала нагрузка на сервер и канал. С учетом того, что в дальнейшем будут использованы более сложные алгоритмы преобразования данных, отображение данных на стороне клиента было решено выполнить в виде растровых изображений. Немного увеличилась нагрузка на канал, однако использование возможностей кэширования растровых изображений на стороне браузера и кэширование растровых изображений после вычислений на стороне сервера при активном применении снизит нагрузку на сервер и веб-сервисы и также позволит просматривать динамику как видеоролик. Пример динамики по времени представлен на нескольких экранных копиях веб-приложения (рис. 3). В результате разработанное веб-приложение отображает всю тематическую информацию либо ее срез в зависимости от настроек пользователя в реальном времени без существенных задержек.

\section{Заключение}

Сегодня в результате развития технологий и программного обеспечения получены новые результаты, которые позволили значительно усовершенствовать существующую программнотехнологическую платформу геопортала ИВМ СО РАН для разработки систем мониторинга окружающей природной среды. Программно-технологическая платформа для организации распределенного доступа к электронной карте Красноярского края позволит усовершенство- 
вать процессы разработки систем мониторинга для Красноярского края, повысит качество предоставляемых услуг для населения края и качество принимаемых управленческих решений. Рассмотренное решение может быть использовано не только для территории Красноярского края, а ресурсы и инструменты разработанной программно-технологической платформы могут быть применены при разработке других систем. Предложенная технологическая основа и карта Красноярского края уже активно используются в различных проектах, работающих в крае.

\section{Список литературы}

[1] Yakubaylik O.E., Kadochnikov A.A., Tokarev A.V. // Russian Digital Libraries Journal, 2014. Vol. 17. Issue 3.

[2] Титов А.Г. , Окладников И.Г. // Вестник НГУ. Информационные технологии. 2014. Т. 12. Вып. 1. С. 79-88.

[3] Якубайлик О.Э., Кадочников А.А., Попов В.Г., Токарев А.В. // Вестник СибГАУ. 2009. Вып. 4 (25). С. 61-66.

[4] Tile Map Service Specification / The Open Source Geospatial Foundation. URL: http://wiki. osgeo.org/wiki/Tile_Map_Service_Specification.

[5] Токарев А.В. // Устойчивое развитие территорий: картографо-геоинформационное обеспечение: материалы Междунар. конф. ИнтерКарто-ИнтерГИС - 20. Белгород: Константа, 2014. C. 228-236.

[6] Информация о состоянии атмосферного воздуха / Подсистема мониторинга атмосферного воздуха. URL: http://krasecology.ru/Air.

[7] Капралов Е.Г., Кошкарев А.В., Тикунов В.С. и др. Основы геоинформатики: учеб. пособие для студ. вузов В 2 кн. Кн. 1: / ред. В.С. Тикунов. М.: Издательский центр «Академия», 2004. $352 \mathrm{c}$.

[8] Sutton T., Dassau O., Sutton M. A Gentle Introduction to GIS. URL: http://download.osgeo. org/qgis/doc/manual/qgis-1.0.0_a-gentle-gis-introduction_en.pdf

[9] Beyer M.A. Gartner Says Solving 'Big Data' Challenge Involves More Than Just Managing Volumes of Data. Gartner. URL: http://www.gartner.com/newsroom/id/1731916. 